\title{
Comparative effectiveness and safety of direct oral anticoagulants versus vitamin $K$ antagonists in nonvalvular atrial fibrillation: a Canadian multicentre observational cohort study
}

\author{
Madeleine Durand MD MSc, Mireille E. Schnitzer PhD, Menglan Pang MSc, Greg Carney PhD, \\ Sherif Eltonsy PhD, Kristian B. Filion PhD, Anat Fisher MD PhD, Min Jun MScMed PhD, \\ I. Fan Kuo PharmD MSc, Christel Renoux MD PhD, J. Michael Paterson MSc, Jacqueline Quail PhD, \\ Alexis Matteau MD MSc; for the Canadian Network for Observational Drug Effect Studies Investigators*
}

\section{Abstract}

Background: Direct oral anticoagulants (DOACs) have widely replaced warfarin for stroke prevention in nonvalvular atrial fibrillation. Our objective was to compare the safety and effectiveness of DOACs (dabigatran, rivaroxaban, apixaban) versus warfarin for stroke prevention in nonvalvular atrial fibrillation in the Canadian setting.

Methods: We conducted a population-based observational multicentre cohort study with propensity score matching and subsequent meta-analysis. We used health care databases from 7 Canadian provinces (British Columbia, Alberta, Saskatchewan, Manitoba, Ontario, Quebec and Nova Scotia). Patients with nonvalvular atrial fibrillation who initiated anticoagulation therapy in 2009-2017 were matched to an equal number who initiated warfarin. The primary outcome was the pooled hazard ratio (HR) for ischemic stroke or systemic embolization. Secondary outcomes included pooled HRs for major bleeding; a composite outcome of stroke, systemic embolization, major bleeding and all-cause mortality; and myocardial infarction. We modelled HRs using proportional hazard Cox regression with inverse probability of censoring weights, and estimated pooled HRs with random-effect meta-analyses.

Results: We included 128273 patients who initiated anticoagulation with a DOAC (40 503 dabigatran, 49498 rivaroxaban and 38272 apixaban) and 128273 patients who initiated anticoagulation with warfarin. The pooled HR for ischemic stroke or systemic embolization comparing DOACs to warfarin was $1.02(95 \%$ confidence interval [CI] 0.87 to 1.19). Direct oral anticoagulants were associated with lower rates of major bleeding (pooled HR 0.81, 95\% $\mathrm{Cl} 0.69$ to 0.97 ), the composite outcome (pooled HR $0.81,95 \%$ $\mathrm{Cl} 0.74$ to 0.89 ) and all-cause mortality (pooled HR $0.81,95 \% \mathrm{Cl} 0.78$ to 0.85 ).

Interpretation: In this real-world study, DOACs were associated with similar risks of ischemic stroke or systemic embolization, and lower risks of bleeding and total mortality compared to warfarin. These findings support the use of DOACs for anticoagulation in nonvalvular atrial fibrillation. Trial registration: ClinicalTrials.gov, no. NCT03596502

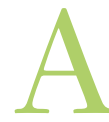

trial fibrillation is the most common type of cardiac arrhythmia, responsible for up to one-third of all strokes. ${ }^{1}$ For most patients with atrial fibrillation, lifelong anticoagulation is indicated to prevent ischemic stroke and systemic arterial embolization. ${ }^{2,3}$ Direct oral anticoagulants (DOACs) are now recommended in clinical care guidelines in Canada, the United States and Europe, as an alternative or in preference to vitamin $\mathrm{K}$ antagonists, in patients with nonvalvular atrial fibrillation., ${ }^{2,4-6}$ In Canada, they have now widely replaced warfarin.

Randomized controlled trials have established noninferiority of DOACs - and superiority in some cases - compared to vitamin $\mathrm{K}$ antagonists with respect to stroke prevention and major bleeding risk. ${ }^{7-10}$ Recent meta-analyses of observational studies showed DOAC treatment to be associated with lower (dabigatran, apixaban) or similar (rivaroxa- ban, edoxaban) bleeding risks, ${ }^{10-14}$ and at least equal effectiveness for stroke prevention ${ }^{10,11,13,14}$ compared to warfarin.

However, there may be important variability in the relative safety and effectiveness of DOACs versus warfarin from one

Competing interests: Min Jun has received unrestricted grant support from VentureWise, a wholly owned subsidiary of NPS MedicineWise funded by AstraZeneca. No other competing interests were declared.

This article has been peer reviewed.

${ }^{*} A$ list of members is provided at the end of the article.

Correspondence to: Madeleine Durand, madeleine.durand.chum@ ssss.gouv.qc.ca

CMAJ Open 2020. DOI:10.9778/cmajo.20200055 
health care system to another. Primary nonadherence and persistence with treatment have been shown to vary widely across countries. ${ }^{15,16}$ A Canadian study using data from Ontario showed about 30\% nonpersistence with dabigatran and rivaroxaban at 6 months, which was associated with increased risks of stroke or transient ischemic attack. ${ }^{17}$ For patients treated with warfarin, time in the therapeutic range for the international normalized ratio has been shown to vary substantially across countries and to affect the safety and effectiveness of warfarin treatment. ${ }^{18-21}$ Data from Canadian jurisdictions are lacking.

We sought to compare the safety and effectiveness of DOACs and warfarin for stroke prevention in nonvalvular atrial fibrillation in the Canadian setting.

\section{Methods}

\section{Study design and setting}

We conducted a matched-cohort study in 7 Canadian provinces (British Columbia, Alberta, Saskatchewan, Manitoba, Ontario, Quebec and Nova Scotia), with each site following a common research protocol, and then meta-analyzed the results across study sites. The study was conducted by the Canadian Network for Observational Drug Effect Studies. ${ }^{22}$

For each site, the study period was defined as the date of availability of dabigatran (the first DOAC to enter the market) until the last date of data availability. The site-specific base cohort entry dates varied from Jan. 1, 2009, to Apr. 24, 2012, and end of follow-up varied from Dec. 31, 2014, to Mar. 31, 2017. The study protocol was registered at ClinicalTrials.gov (NCT03596502).

\section{Data sources}

We used administrative health care data from the 7 provinces. All sites had access to provincial health insurance registries, physician billing claims, emergency department records (except for BC, Manitoba and Quebec), hospital discharge abstracts (medical diagnoses) and prescription drug claims. The Ontario and Nova Scotia data were restricted to patients aged 65 years or more. The Quebec data were restricted to those aged 65 years or more, beneficiaries of social assistance and those subscribing to the public insurance drug plan (40\% of the total population). Other jurisdictions had no age or social status limitations. The sites and their data sources have been described in detail previously. ${ }^{22}$ The names of the individual data sets are listed in Appendix 1 (available at www. cmajopen.ca/content/8/4/E877/suppl/DC1). These data sets are linked in an anonymous fashion by means of coded health insurance numbers, they have very little missing information, ${ }^{23-25}$ and they are used routinely to study drug safety. ${ }^{26-28}$

\section{Participants}

At each site, we created a base cohort of all patients aged 18 years or more who initiated oral anticoagulant therapy and had a diagnosis of nonvalvular atrial fibrillation. We defined the date of the first dispensation for warfarin, dabigatran, rivaroxaban or apixaban as base cohort entry date. Patients were excluded if they had received oral anticoagulant treatment with any of the study drugs in the prior 365 days. Other exclusion criteria (applied sequentially) were absence of health care coverage in the 365 days before entry date; diagnosis of venous thromboembolism in the 365 days before entry date; hemodialysis in the 90 days before entry date; diagnosis of valvular heart disease or cardiac valve surgery at any time before entry date; hip, femur or knee surgery within 30 days before entry date; a diagnosis of antiphospholipid syndrome at any time in the past; and an absence of a diagnostic code for atrial fibrillation or atrial flutter in the prior 3 years (International Classification of Diseases, 9th Revision [ICD-9] 427.3, International Statistical Classification of Diseases and Related Health Problems, 10th Revision [ICD-10] I48.x). Patients were followed until occurrence of an event (defined below), censoring owing to death, end of health or drug insurance, or end of data availability, whichever occurred first.

\section{Study cohort definition}

From the base cohort at each participating site, we assembled a study cohort of new users of orally administered anticoagulants comparing DOACs to warfarin. Each patient entering the base cohort with a dispensation for a DOAC was matched with replacement to 1 patient entering the base cohort with warfarin. Patients were matched on age at cohort entry date $( \pm 365 \mathrm{~d}$ if exact birthdate was known, $\pm 1 \mathrm{yr}$ if age was rounded to the year, or in the same age category for sites with age categories), sex, study cohort entry date $( \pm 365 \mathrm{~d})$ and a propensity score measuring the probability of receiving DOACs (within a maximum propensity score caliper of $0.2 *$ standard deviation of the propensity score on the logit scale). Users of DOACs with no possible matches were excluded.

We modelled propensity to receive DOACs at base cohort entry using logistic regression conditional on baseline covariates, which we selected based on a priori knowledge. These included age, sex, all components of the CHADS (congestive heart failure, hypertension, age $\geq 75 \mathrm{yr}$, diabetes, stroke) score $^{29}$ and HAS-BLED (hypertension, abnormal renal and liver function, stroke, bleeding, labile international normalized ratio, elderly [age $>65 \mathrm{yr}$ ], drugs or alcohol [ $\geq 8$ drinks/wk]) score, ${ }^{30}$ and other medical comorbidities, procedures or drugs deemed to be associated with stroke or bleeding risk. All included covariates are listed in Appendix 2 (available at www. cmajopen.ca/content/8/4/E877/suppl/DC1). We trimmed propensity score distributions at the lowest and highest $5 \%$ of the propensity score values (over both groups together).

\section{Study period and exposure definition}

We defined cohort entry date as the date of base cohort entry (date of first dispensation for oral anticoagulant therapy) for both DOAC users and warfarin users. Patients were rightcensored at the earliest of end of health insurance coverage, end of data availability, switch from warfarin to DOAC, switch from DOAC to warfarin or occurrence of an absolute contraindication to DOAC therapy (defined as initiation of hemodialysis or heart valve surgery). Patients were considered exposed to the anticoagulant received at baseline until censored. 


\section{Outcomes}

The primary outcome was ischemic stroke or systemic embolization defined in an acute care hospital discharge abstract and labelled as the primary or most responsible diagnosis for the admission. Ischemic stroke and systemic embolization were defined with ICD-9 (434.x, 444.x) and ICD-10 (I63.x, I64.x, I74.x) codes for all sites.

The prespecified secondary outcomes were major bleeding; a composite outcome of stroke (ischemic or hemorrhagic), systemic embolization, major bleeding and all-cause mortality; myocardial infarction; and composite outcome stratified by age ( $<85 \mathrm{v} . \geq 85 \mathrm{yr}$ at cohort entry date) and sex. We defined major bleeding as a composite of intracranial (including hemorrhagic stroke), gastrointestinal, ocular, and any other bleeding necessitating hospital admission or an emergency department visit. The complete list of diagnostic codes used to define the secondary outcomes is provided in Appendix 2.

\section{Statistical analysis}

We described baseline characteristics of patients in the base and study cohorts using means and proportions.

For each study site, we constructed Cox proportional hazard models with censoring weights to model the hazard ratios (HRs) for each outcome. The models did not account for matching. ${ }^{31}$ To account for nonrandom attrition due to switches from warfarin to a DOAC or from a DOAC to warfarin, we used inverse probability of censoring weights. ${ }^{32,33}$ We built weights as follows. Patient covariates, treatment use and censoring status were updated at 28-day intervals. Then, we fit a pooled logistic regression model across all intervals, which modelled the probability of remaining uncensored at each time $t$ given the covariates at time $t-1$. Weights used in the analysis were the inverse of that probability. We generated censoring weights (1 per at-risk patient-time) separately (in fully stratified models) for DOAC and warfarin initiators. We did this because a switch from a DOAC to warfarin is due to a different clinical dynamic than a switch from warfarin to a DOAC. For example, patients with chronic renal disease are more likely to switch from a DOAC to warfarin and less likely to switch from warfarin to a DOAC.

To estimate the incidence of the components of the primary and secondary outcomes, we generated survival curves and derived the corresponding 1-year cumulative incidence proportion from the Kaplan-Meier estimates.

To study effect modification by age and sex, we added to the models an interaction term between treatment group and age less than 85 years and age 85 years or more, and between treatment group and sex, and presented stratified results. We assessed the significance of the interaction term using a likelihood ratio test. We conducted site-specific analyses using SAS software (SAS Institute).

We meta-analyzed marginal site-specific HRs, 1-year cumulative incidence proportions and incidence differences for each of the primary and secondary outcomes across sites using DerSimonian and Laird random-effects models with inverse variance weighting to estimate pooled HRs and their $95 \%$ confidence intervals (CIs). We chose random-effects models a priori owing to the heterogeneity of populations across sites. We calculated the $I^{2}$ statistic to describe heterogeneity. We performed meta-analyses using the metan command in Stata version 14.1 (StataCorp).

\section{Ethics approval}

Research ethics board approval was obtained at participating sites as required (Appendix 1).

\section{Results}

Patients' selection into the base cohort is shown in Figure 1. At base cohort entry, 142336 patients received DOACs (of whom 44639 [31.4\%] received dabigatran, 55131 [38.7\%] rivaroxaban and 42566 [29.9\%] apixaban), and 113515 received warfarin. The baseline characteristics of patients included in the base cohort are presented in Table 1 and Appendix 3, Supplemental Table S1 (available at www. cmajopen.ca/content/8/4/E877/suppl/DC1). Compared to patients receiving DOACs, those receiving warfarin were older (75.8 v. $74.7 \mathrm{yr}$ ) and more likely to have medical comorbidities such as congestive heart failure $(34.2 \%$ v. $23.6 \%)$, hypertension $(80.1 \%$ v. $74.6 \%)$, diabetes $(36.4 \%$ v. $30.1 \%)$, prior stroke (20.6\% v. $16.5 \%)$, chronic kidney injury $(18.0 \%$ v. $10.1 \%)$ and peripheral vascular disease (18.2\% v. $12.6 \%)$.

The characteristics of the study cohort are presented in Table 2 and Appendix 3, Supplemental Table S2. Among the patients initiating DOACs, we were able to match 128273 $(90.1 \%)$ to a patient initiating warfarin. Of the DOAC initiators, 40503 (31.6\%) took dabigatran, 49498 (38.6\%) took rivaroxaban and $38272(29.8 \%)$ took apixaban. The DOAC and warfarin groups were similar in mean age (75.3 yr), sex $(52.4 \%$ male) and all covariates included in the propensity score, including CHADS score (mean 2.4 in the 2 groups). The mean total length of follow-up was 2 years for patients initiating warfarin and 1.9 years for those initiating a DOAC. During follow-up, $5.7 \%$ of DOAC initiators switched to warfarin, and $35.0 \%$ of warfarin initiators switched to a DOAC. Among DOACs initiators, the lower dosing regimen was received by $51.5 \%$ of dabigatran users, $30.3 \%$ of rivaroxaban users and $35.1 \%$ of apixaban users.

The results of the analysis for the primary and secondary outcomes are presented in Table 3, Figure 2 and Appendix 3, Supplemental Figure S1. There was no difference in the pooled HR for ischemic stroke or systemic embolization between DOACs and warfarin (pooled HR 1.02, 95\% CI 0.87 to 1.19$)$, with no evidence of significant heterogeneity across sites $\left(I^{2}=25.6 \%, p=0.2\right)$. There were lower risks of major bleeding (pooled HR 0.81, 95\% CI 0.69 to 0.97 ) and intracranial bleeding (pooled HR 0.55 , 95\% CI 0.45 to 0.66 ) with DOACs than with warfarin. There was no difference in pooled HR for myocardial infarction or gastrointestinal bleeding. The pooled HR for the composite outcome of stroke, systemic embolization, major bleeding and all-cause mortality favoured DOAC (pooled HR 0.81, 95\% CI 0.74 to 0.89 ). Allcause mortality was lower in the DOAC group (pooled HR $0.81,95 \%$ CI 0.78 to 0.85$)$. Similar results were observed 


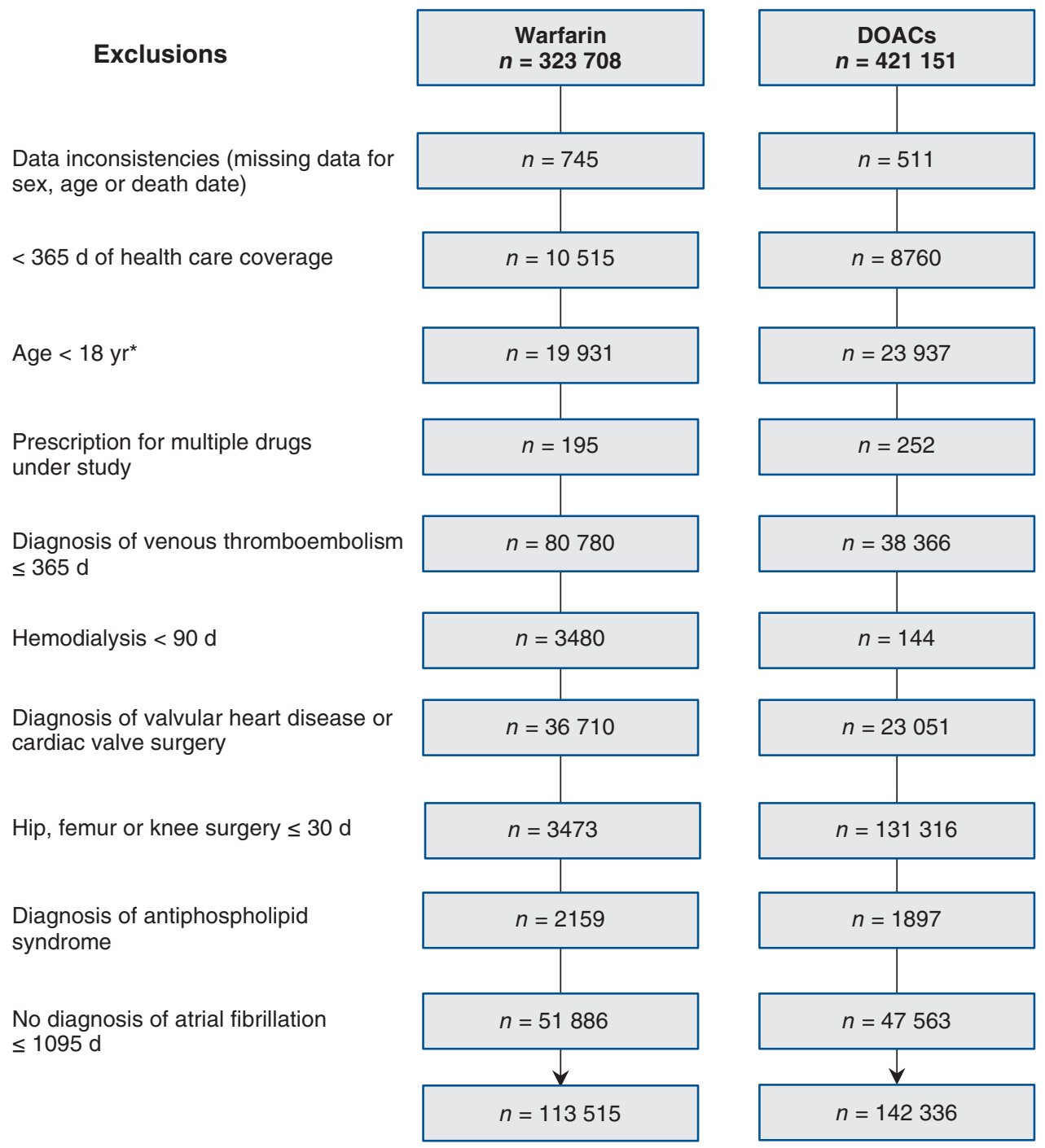

Figure 1: Flow diagram showing inclusion of patients into the base cohort. Numbers do not add up exactly owing to suppression of small cells for confidentiality reasons. Note: DOAC = direct oral anticoagulant. *Less than 66 years for sites with comprehensive drug claim data only for older adults (Ontario, Nova Scotia).

across subgroups defined by age and sex (Appendix 3, Supplemental Table S3). The absolute number of events for each outcome are shown in Appendix 3, Supplemental Table S4. For the outcomes of major bleeding and the composite outcome, there was substantial heterogeneity in point estimates across sites.

\section{Cumulative 1-year incidence of outcomes}

Table 4 and Appendix 3, Supplemental Figure S2 show the 1 -year cumulative incidences and corresponding incidence differences for each outcome. At 1 year, exposure to DOACs compared to warfarin was associated with -1.56 (95\% CI -2.79 to -0.33 ) ischemic strokes or systemic emboli per 1000 patient-years. Exposure to DOACs was associated with
-6.16 (95\% CI -13.55 to 1.22 ) major bleeding events, -1.25 (95\% CI -2.32 to -0.17 ) intracranial bleeds and -13.79 (95\% CI -21.59 to -5.98$)$ deaths per 1000 patients at 1 year.

\section{Interpretation}

We found no difference in the incidence of ischemic stroke or systemic embolization in patients with nonvalvular atrial fibrillation who received anticoagulation therapy with DOACs versus warfarin. However, we found that use of DOACs was associated with fewer major bleeding events, intracranial bleeding events and deaths.

Our findings are largely consistent with the published literature. Recent meta-analyses of observational studies showed 


\begin{tabular}{|c|c|c|}
\hline \multirow[b]{2}{*}{ Characteristic } & \multicolumn{2}{|c|}{ Group; no. (\%) of patients ${ }^{*} \dagger$} \\
\hline & $\begin{array}{c}\text { DOAC } \\
n=142336\end{array}$ & $\begin{array}{c}\text { Warfarin } \\
n=113515\end{array}$ \\
\hline Age, mean $\pm S E, y r$ & $74.7 \pm 1.3$ & $75.8 \pm 0.9$ \\
\hline Sex, male & $76572(53.8)$ & $61200(53.9)$ \\
\hline \multicolumn{3}{|l|}{ Year of base cohort entry } \\
\hline 2009-2010 & $302(0.2)$ & $19093(16.8)$ \\
\hline $2011-2012$ & $29959(21.0)$ & $40022(35.3)$ \\
\hline 2013-2014 & $56574(39.7)$ & $37457(33.0)$ \\
\hline $2015-2016$ & $55420(38.9)$ & $16943(14.9)$ \\
\hline \multicolumn{3}{|l|}{ DOAC received at study entry } \\
\hline Dabigatran & $44639(31.4)$ & - \\
\hline 110 mg twice daily & $22296(49.9)$ & - \\
\hline 150 mg twice daily & $20409(45.7)$ & - \\
\hline Other & $1934(4.3)$ & - \\
\hline Rivaroxaban & $55131(38.7)$ & - \\
\hline $15 \mathrm{mg}$ once daily & $13473(24.4)$ & - \\
\hline 20 mg once daily & $39005(70.7)$ & - \\
\hline Other & $2653(4.8)$ & - \\
\hline Apixaban & $42566(29.9)$ & - \\
\hline $2.5 \mathrm{mg}$ twice daily & $14250(33.5)$ & - \\
\hline $5 \mathrm{mg}$ twice daily & $26504(62.3)$ & - \\
\hline Other & $1812(4.3)$ & - \\
\hline Treatment switches & $6826(4.8)$ & $32780(28.9)$ \\
\hline Length of follow-up, mean $\pm S E, d$ & $624.7 \pm 67.1$ & $1015.2 \pm 96.1$ \\
\hline \multicolumn{3}{|l|}{ Medical diagnoses } \\
\hline Congestive heart failure & $33558(23.6)$ & $38824(34.2)$ \\
\hline Hypertension & $106256(74.6)$ & $90957(80.1)$ \\
\hline Diabetes & $42813(30.1)$ & $41327(36.4)$ \\
\hline Stroke & $23540(16.5)$ & $23396(20.6)$ \\
\hline Transient ischemic attack & $7314(5.1)$ & $6788(6.0)$ \\
\hline Chronic kidney injury & $14345(10.1)$ & $20470(18.0)$ \\
\hline Acute kidney injury & $5798(4.1)$ & $9505(8.4)$ \\
\hline Liver disease & $5739(4.0)$ & $4270(3.8)$ \\
\hline Cancer & $15039(10.6)$ & $14606(12.9)$ \\
\hline Chronic obstructive pulmonary disease & $38010(26.7)$ & $38864(34.2)$ \\
\hline Coronary atherosclerosis & $64340(45.2)$ & $57846(51.0)$ \\
\hline Myocardial infarction & $19978(14.0)$ & 21914 (19.3) \\
\hline Peripheral vascular disease & $17873(12.6)$ & $20668(18.2)$ \\
\hline Prior bleeding & $9208(6.5)$ & $11942(10.5)$ \\
\hline Dementia & $14866(10.4)$ & $12018(10.6)$ \\
\hline
\end{tabular}




\begin{tabular}{|c|c|c|}
\hline \multirow[b]{2}{*}{ Characteristic } & \multicolumn{2}{|c|}{ Group; no. (\%) of patients $†$} \\
\hline & $\begin{array}{c}\text { DOAC } \\
n=128273\end{array}$ & $\begin{array}{c}\text { Warfarin } \\
n=128273\end{array}$ \\
\hline Age, mean $\pm S E, y r$ & $75.3 \pm 0.9$ & $75.3 \pm 0.9$ \\
\hline Sex, male & $67159(52.4)$ & $67159(52.4)$ \\
\hline \multicolumn{3}{|l|}{ Year of base cohort entry } \\
\hline 2009-2010 & $259(0.2)$ & $1222(1.0)$ \\
\hline $2011-2012$ & $27332(21.3)$ & $26356(20.5)$ \\
\hline 2013-2014 & $51343(40.0)$ & 55451 (43.2) \\
\hline $2015-2016$ & $49339(38.5)$ & $45244(35.3)$ \\
\hline \multicolumn{3}{|l|}{ DOAC received at study entry } \\
\hline Dabigatran & $40503(31.6)$ & - \\
\hline $110 \mathrm{mg}$ twice daily & $20857(51.5)$ & - \\
\hline $150 \mathrm{mg}$ twice daily & $18134(44.8)$ & - \\
\hline Other & $1512(3.7)$ & - \\
\hline Rivaroxaban & $49498(38.6)$ & - \\
\hline $15 \mathrm{mg}$ once daily & $14981(30.3)$ & - \\
\hline 20 mg once daily & $31706(64.1)$ & - \\
\hline Other & $2811(5.7)$ & - \\
\hline Apixaban & $38272(29.8)$ & - \\
\hline $2.5 \mathrm{mg}$ twice daily & $13452(35.1)$ & - \\
\hline $5 \mathrm{mg}$ twice daily & $23465(61.3)$ & - \\
\hline Other & $1355(3.5)$ & - \\
\hline Treatment switches & $7360(5.7)$ & $44950(35.0)$ \\
\hline Length of follow-up, mean $\pm S E, d$ & $715 \pm 50.20$ & $762 \pm 93.3$ \\
\hline \multicolumn{3}{|l|}{ Medical diagnoses } \\
\hline Congestive heart failure & $29970(23.4)$ & $29740(23.2)$ \\
\hline Hypertension & 99835 (77.8) & $99430(77.5)$ \\
\hline Diabetes & $38801(30.2)$ & $38931(30.4)$ \\
\hline Stroke & $22274(17.4)$ & $22178(17.3)$ \\
\hline Transient ischemic attack & $6557(5.1)$ & $6354(5.0)$ \\
\hline Chronic kidney injury & $11423(8.9)$ & $12391(9.7)$ \\
\hline Acute kidney injury & $3843(3.0)$ & 4961 (3.9) \\
\hline Liver disease & $4882(3.8)$ & $5196(4.0)$ \\
\hline Cancer & $13624(10.6)$ & $14186(11.1)$ \\
\hline Chronic obstructive pulmonary disease & $34308(26.7)$ & $34553(26.9)$ \\
\hline Coronary atherosclerosis & $56766(44.2)$ & $54435(42.4)$ \\
\hline Myocardial infarction & $17587(13.7)$ & $17714(13.8)$ \\
\hline Peripheral vascular disease & $15997(12.5)$ & $16526(12.9)$ \\
\hline Prior bleeding & $8115(6.3)$ & $8912(6.9)$ \\
\hline Dementia & 12691 (9.9) & $12758(9.9)$ \\
\hline CHADS score, mean \pm SE & $2.41 \pm 0.43$ & $2.4 \pm 0.41$ \\
\hline \multicolumn{3}{|c|}{$\begin{array}{l}\text { Note: } \mathrm{CHADS}=\text { congestive heart failure, hypertension, age } \geq 75 \mathrm{yr} \text {, diabetes, stroke; } \mathrm{DOAC}=\text { direct oral } \\
\text { anticoagulant; } \mathrm{SE}=\text { standard error. } \\
{ }^{*} \text { Matched on age, sex, calendar date and propensity score. } \\
\text { †Except where noted otherwise. }\end{array}$} \\
\hline
\end{tabular}

either a similar or a lower risk of stroke associated with DOAC use compared to warfarin, with heterogeneity regarding which DOAC was associated with reduced risk of stroke. ${ }^{10,13,14}$ A large observational study from Sweden, in which warfarin-exposed patients had a time in therapeutic range of international normalized ratio measurements of 


\begin{tabular}{|c|c|c|}
\hline \multirow[b]{2}{*}{ Outcome } & \multicolumn{2}{|c|}{$\mathrm{HR}(95 \% \mathrm{Cl})$} \\
\hline & Crude* & Adjusted $\dagger$ \\
\hline Ischemic stroke or systemic embolization & 0.88 (0.80 to 0.97$)$ & $1.02(0.87$ to 1.20$)$ \\
\hline Major bleeding & 0.81 (0.72 to 0.92$)$ & 0.81 (0.69 to 0.97$)$ \\
\hline $\begin{array}{l}\text { Composite of ischemic stroke or systemic embolization, } \\
\text { major bleeding, and all-cause mortality }\end{array}$ & $0.78(0.73$ to 0.84$)$ & $0.81(0.74$ to 0.89$)$ \\
\hline Myocardial infarction & $0.92(0.83$ to 1.02$)$ & $0.96(0.84$ to 1.09$)$ \\
\hline Intracranial bleeding & $0.62(0.54$ to 0.71$)$ & $0.55(0.45$ to 0.66$)$ \\
\hline Gastrointestinal bleeding & $0.98(0.89$ to 1.07$)$ & $1.00(0.88$ to 1.15$)$ \\
\hline All-cause mortality & $0.74(0.70$ to 0.78$)$ & $0.81(0.78$ to 0.85$)$ \\
\hline $\begin{array}{l}\text { Note: } \mathrm{Cl}=\text { confidence interval, } \mathrm{HR} \text { = hazard ratio. } \\
\text { *Unadjusted for censoring. } \\
\text { †Weighted model for inverse probability of censoring. }\end{array}$ & & \\
\hline
\end{tabular}

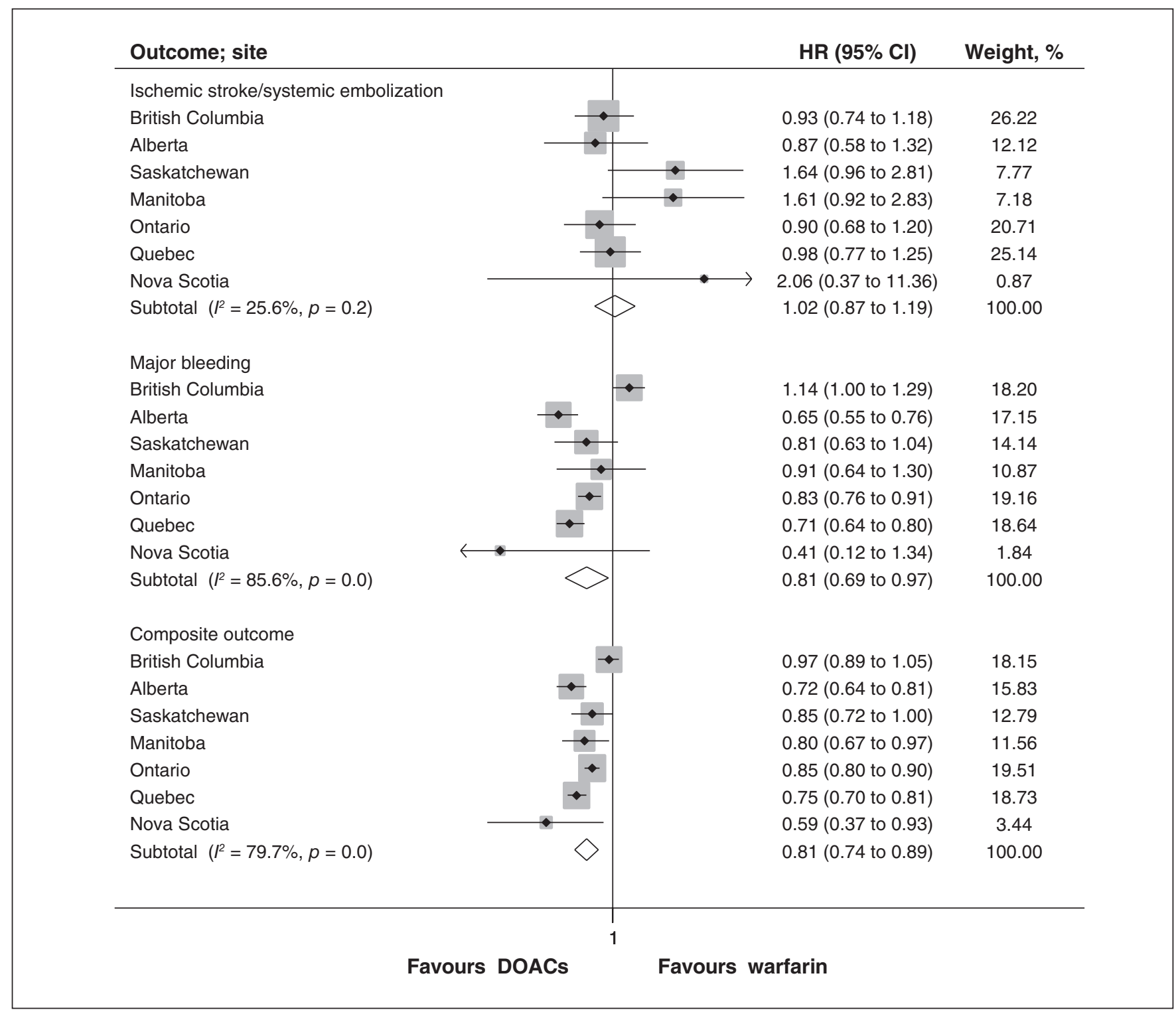

Figure 2: Pooled hazard ratios (HRs) for stroke or systemic embolization, major bleeding, and the composite outcome of stroke, systemic embolization, major bleeding and all-cause mortality. Note: $\mathrm{Cl}=$ confidence interval. 
Table 4: Pooled cumulative incidence and incidence difference per 1000 patients at 1 year for individual components of the primary and secondary outcomes

Group; 1-year cumulative incidence per 1000 patients $(95 \% \mathrm{Cl})$

\begin{tabular}{|c|c|c|c|}
\hline \multirow[b]{2}{*}{ Outcome } & & \multirow{2}{*}{$\begin{array}{l}\text { Incidence difference } \\
\qquad(95 \% \mathrm{Cl})\end{array}$} \\
\hline & DOAC & Warfarin & \\
\hline $\begin{array}{l}\text { Ischemic stroke or systemic } \\
\text { embolization }\end{array}$ & 7.71 (5.67 to 9.75$)$ & 8.88 (5.22 to 12.54$)$ & $-1.56(-2.79$ to -0.33$)$ \\
\hline Major bleeding & 35.97 (23.60 to 48.34$)$ & 41.86 (21.23 to 62.49$)$ & $-6.16(-13.55$ to 1.22$)$ \\
\hline Intracranial bleeding & $2.84(1.87$ to 3.81$)$ & 4.10 (1.58 to 6.62$)$ & $-1.25(-2.32$ to -0.17$)$ \\
\hline Gastrointestinal bleeding & $19.00(14.11$ to 23.90$)$ & 15.49 (8.99 to 21.98$)$ & $1.08(-0.72$ to 2.88$)$ \\
\hline Myocardial infarction & 7.50 (5.55 to 9.36$)$ & 7.40 (3.47 to 11.32$)$ & $-0.29(-1.42$ to 0.83$)$ \\
\hline All-cause mortality & 54.00 (37.91 to 70.09$)$ & 65.64 (47.16 to 84.12$)$ & $-13.79(-21.59$ to -5.98$)$ \\
\hline
\end{tabular}

71.4\%, also showed a similar HR for ischemic strokes for DOACs and warfarin. ${ }^{34}$ In their recent observational studies, however, both Lip and colleagues ${ }^{35}$ and Graham and colleagues $^{36}$ reported decreased risk of stroke with all 3 DOACs compared to warfarin.

Recent meta-analyses showed similar major bleeding risks with rivaroxaban compared to warfarin, ${ }^{10,11,13,14}$ lower or similar major bleeding risk with dabigatran, ${ }^{10,11,13}$ and lower major bleeding risk with apixaban. ${ }^{10,11,13}$ The finding that is most consistent across the literature is that of lower intracranial bleeds with DOACs than with warfarin. ${ }^{11,13,14,36,37}$ Increased mortality in warfarin cohorts was also reported in a network meta-analysis of 18 randomized controlled trials, as well as in a large recent observational study. ${ }^{36,38}$ Our study confirms that use of DOACs confers similar results in terms of safety and effectiveness in the Canadian setting compared to other jurisdictions.

We found substantial heterogeneity across provinces in the composite and safety outcomes. This may have been due to the distinct populations captured in the data (e.g., Ontario captures data only for people aged $\geq 65 \mathrm{yr}$, Quebec captures a fraction of younger people, and the other provinces' databases capture all ages). It may also have been due to different practices in use of warfarin or DOACs that our data did not capture.

Strengths of our study include its large sample and the inclusion of data from 7 provinces, which make our results generalizable to the Canadian population. The new user design is another strength, as it avoids bias due to inclusion of treatment switchers and bias due to depletion of susceptibles, which are present when patients previously exposed to warfarin are included as new users of DOACs. ${ }^{39}$ The use of a common protocol to conduct the studies in each of the participating sites also ensured better comparability of the results across sites and meaningful pooled estimates. Our exposure definition is reliable, as it is based on pharmaceutical dispensations.

Our outcome definitions relied on hospital discharge abstracts, which are reviewed by trained medical record abstractors and therefore are considered a reliable data source. The Canadian Institute for Health Information performed an extensive review of the validity of abstract discharge summaries across Canada and found high agreement between admission diagnoses in the discharge abstract database and reabstraction by chart review. For instance, the agreement for ischemic stroke yielded a $\kappa$ value of 0.81 (95\% CI 0.77 to 0.85 ), sensitivity of $76 \%$ (95\% CI $70 \%$ to $81 \%$ ) and a positive predictive value of $87 \%$ (95\% CI $82 \%$ to $91 \%){ }^{25}$

\section{Limitations}

We tried to minimize confounding by matching on a propensity score that adjusted for an extensive list of medical comorbidities, medical procedures and co-medications, and ensured that potential informative censoring was adjusted for with the use of inverse probability of censoring weights. Nevertheless, several potential confounders, such as socioeconomic status, smoking and, most important of all, precise measures of kidney function, were absent from the data. Our results may be subject to residual confounding, which would have biased results in favour of the DOAC group, as DOACs are contraindicated at various levels of renal failure. Our data also lacked international normalized ratio measurements; therefore, we were unable to calculate time in therapeutic target for the patients treated with warfarin. We also performed numerous analyses given the large number of secondary outcomes. We recognize that this may have led to inflation of type 1 error and that the results for secondary outcomes should be interpreted with caution.

\section{Conclusion}

We found that the use of DOACs in patients with nonvalvular atrial fibrillation in the Canadian setting was associated with similar protection from ischemic stroke and systemic embolization compared to warfarin, as well as less major bleeding, in particular intracranial bleeding. These findings support the use of DOACs for anticoagulation in nonvalvular atrial fibrillation. 


\section{References}

1. Atrial fibrillation. Ottawa: Heart and Stroke Foundation. Available: www. heartandstroke.ca/heart-disease/conditions/atrial-fibrillation (accessed 2020 Dec. 10).

2. Verma A, Cairns JA, Mitchell LB, et al.; CCS Atrial Fibrillation Guidelines Committee. 2014 focused update of the Canadian Cardiovascular Society Guidelines for the management of atrial fibrillation. Can 7 Cardiol 2014;30: 1114-30.

3. National Clinical Guideline Centre (UK). Atrial fibrillation: the management of atrial fibrillation. London (UK): National Institute for Health and Care Excellence; 2014.

4. January CT, Wann LS, Calkins H, et al. 2019 AHA/ACC/HRS focused update of the 2014 AHA/ACC/HRS guideline for the management of patients with atrial fibrillation. Circulation 2019;140:e125-51.

5. Andrade JG, Verma A, Mitchell LB, et al. 2018 focused update of the Canadian Cardiovascular Society guidelines for the management of atrial fibrillation. Can 7 Cardiol 2018;34:1371-92.

6. Kirchhof P, Benussi S, Kotecha D, et al.; ESC Scientific Document Group. 2016 ESC guidelines for the management of atrial fibrillation developed in collaboration with EACTS. Eur Heart 7 2016;37:2893-962.

7. Patel MR, Mahaffey KW, Garg J, et al.; ROCKET AF Investigators. Rivaroxaban versus warfarin in nonvalvular atrial fibrillation. N Engl $\mathcal{7}$ Med 2011;365: 883-91.

8. Connolly SJ, Ezekowitz MD, Yusuf S, et al.; RE-LY Steering Committee and Investigators. Dabigatran versus warfarin in patients with atrial fibrillation. $N$ Engl 7 Med 2009;361:1139-51

9. Granger CB, Alexander JH, McMurray JJ, et al.; ARISTOTLE Committees and Investigators. Apixaban versus warfarin in patients with atrial fibrillation. $N$ Engl7 Med 2011;365:981-92.

10. Lowenstern A, Al-Khatib SM, Sharan L, et al. Interventions for preventing thromboembolic events in patients with atrial fibrillation: a systematic review. Ann Intern Med 2018;169:774-87.

11. Deitelzweig S, Farmer C, Luo X, et al. Risk of major bleeding in patients with non-valvular atrial fibrillation treated with oral anticoagulants: a systematic review of real-world observational studies. Curr Med Res Opin 2017;33: 1583-94.

12. Hirschl M, Kundi M. Safety and efficacy of direct acting oral anticoagulants and vitamin $\mathrm{K}$ antagonists in nonvalvular atrial fibrillation - a network metaanalysis of real-world data. Vasa 2019;48:134-47.

13. Escobar C, Martí-Almor J, Pérez Cabeza A, et al. Direct oral anticoagulants versus vitamin $\mathrm{K}$ antagonists in real-life patients with atrial fibrillation. A systematic review and meta-analysis. Rev Esp Cardiol (Engl Ed) 2019;72:305-16.

14. Bai Y, Deng H, Shantsila A, et al. Rivaroxaban versus dabigatran or warfarin in real-world studies of stroke prevention in atrial fibrillation: systematic review and meta-analysis. Stroke 2017;48:970-6.

15. Rodriguez-Bernal CL, Peiró S, Hurtado I, et al. Primary nonadherence to oral anticoagulants in patients with atrial fibrillation: real-world data from a population-based cohort. 7 Manag Care Spec Pharm 2018;24:440-8.

16. Hylek EM. Treatment persistence in atrial fibrillation: the next major hurdle. Thromb Haemost 2018;118:2018-9.

17. Jackevicius CA, Tsadok MA, Essebag V, et al. Early non-persistence with dabigatran and rivaroxaban in patients with atrial fibrillation. Heart 2017;103: 1331-8.

18. Amin A, Deitelzweig S, Jing Y, et al. Estimation of the impact of warfarin's time-in-therapeutic range on stroke and major bleeding rates and its influence on the medical cost avoidance associated with novel oral anticoagulant use learnings from ARISTOTLE, ROCKET-AF, and RE-LY trials. 7 Thromb Thrombolysis 2014;38:150-9.

19. Connolly SJ, Pogue J, Eikelboom J, et al.; ACTIVE W Investigators. Benefit of oral anticoagulant over antiplatelet therapy in atrial fibrillation depends on the quality of international normalized ratio control achieved by centers and countries as measured by time in therapeutic range. Circulation 2008;118: 2029-37.

20. Wallentin L, Yusuf S, Ezekowitz MD, et al.; RE-LY investigators. Efficacy and safety of dabigatran compared with warfarin at different levels of international normalised ratio control for stroke prevention in atrial fibrillation: an analysis of the RE-LY trial. Lancet 2010;376:975-83

21. McAlister FA, Wiebe N, Hemmelgarn BR. Time in therapeutic range and stability over time for warfarin users in clinical practice: a retrospective cohort study using linked routinely collected health data in Alberta, Canada. BMF Open 2018;8:e016980.

22. Suissa S, Henry D, Caetano P, et al.; Canadian Network for Observational Drug Effect Studies (CNODES). CNODES: the Canadian Network for Observational Drug Effect Studies. Open Med 2012;6:e134-40.

23. Levy AR, O'Brien BJ, Sellors C, et al. Coding accuracy of administrative drug claims in the Ontario Drug Benefit database. Can 7 Clin Pharmacol 2003;10: 67-71.

24. Williams J, Young W. Appendix I: A summary of studies on the quality of health care administrative databases in Canada. In: Goel V, Williams JI, Anderson GM, et al., editors. Patterns of health care in Ontario. The ICES Practice Atlas. 2nd ed. Ottawa: Canadian Medical Association; 1996:339.
25. Juurlink D, Preyra C, Croxford R, et al. Canadian Institute for Health Information Discharge Abstract Database: a validation study. Toronto: ICES; 2006.

26. Filion KB, Azoulay L, Platt RW, et al. A multicenter observational study of incretin-based drugs and heart failure. N Engl 7 Med 2016;374:1145-54.

27. Renoux C, Lix LM, Patenaude V, et al. Serotonin-norepinephrine reuptake inhibitors and the risk of AKI: a cohort study of eight administrative databases and meta-analysis. Clin 7 Am Soc Nephrol 2015;10:1716-22.

28. Dormuth CR, Filion KB, Paterson JM, et al.; Canadian Network for Observational Drug Effect Studies Investigators. Higher potency statins and the risk of new diabetes: multicentre, observational study of administrative databases. BM7 2014;348:g3244.

29. Gage BF, Waterman AD, Shannon $W$, et al. Validation of clinical classification schemes for predicting stroke: results from the National Registry of Atrial Fibrillation. $7 A M A$ 2001;285:2864-70.

30. Apostolakis S, Lane DA, Guo Y, et al. Performance of the HEMORR 2 HAGES, ATRIA, and HAS-BLED bleeding risk-prediction scores in patients with atrial fibrillation undergoing anticoagulation: the AMADEUS (Evaluating the Use of SR34006 Compared to Warfarin or Acenocoumarol in Patients With Atrial Fibrillation) study. 7 Am Coll Cardiol 2012;60:861-7.

31. Hill J, Reiter JP. Interval estimation for treatment effects using propensity score matching. Stat Med 2006;25:2230-56.

32. Robins JM, Finkelstein DM. Correcting for noncompliance and dependent censoring in an AIDS clinical trial with inverse probability of censoring weighted (IPCW) log-rank tests. Biometrics 2000;56:779-88.

33. Robins JM, Hernán MA, Brumback B. Marginal structural models and causal inference in epidemiology. Epidemiology 2000;11:550-60.

34. Själander S, Sjögren V, Renlund H, et al. Dabigatran, rivaroxaban and apixaban vs. high TTR warfarin in atrial fibrillation. Thromb Res 2018;167:113-8.

35. Lip GYH, Keshishian A, Li X, et al. Effectiveness and safety of oral anticoagulants among nonvalvular atrial fibrillation patients. Stroke 2018;49:2933-44.

36. Graham DJ, Baro E, Zhang R, et al. Comparative stroke, bleeding, and mortality risks in older Medicare patients treated with oral anticoagulants for nonvalvular atrial fibrillation. Am $\mathcal{7}$ Med 2019;132:596-604.e11.

37. Vinogradova Y, Coupland C, Hill T, et al. Risks and benefits of direct oral anticoagulants versus warfarin in a real world setting: cohort study in primary care. $B M 72018 ; 362: \mathrm{k} 2505$.

38. Antza C, Doundoulakis I, Akrivos E, et al. Non-vitamin K oral anticoagulants in nonvalvular atrial fibrillation: a network meta-analysis. Scand Cardiovasc 7 2019;53:48-54.

39. Hernán MA. Counterpoint: epidemiology to guide decision-making: moving away from practice-free research. Am $\mathcal{F}$ Epidemiol 2015;182:834-9.

Affiliations: Internal Medicine Service (Durand), Centre hospitalier de l'Université de Montréal (CHUM) and CHUM Research Centre (Durand); Faculty of Pharmacy (Schnitzer) and Department of Social and Preventive Medicine (Schnitzer), Université de Montréal; Departments of Epidemiology, Biostatistics and Occupational Health (Schnitzer, Pang, Filion, Renoux), Medicine (Filion) and Neurology and Neurosurgery (Renoux), McGill University, Montréal, Que.; Departments of Anesthesiology, Pharmacology and Therapeutics (Carney, Fisher), Faculty of Medicine, University of British Columbia, Vancouver, BC; College of Pharmacy (Eltonsy, Kuo), Rady Faculty of Health Sciences, University of Manitoba, Winnipeg, Man.; Centre for Clinical Epidemiology (Filion, Renoux), Lady Davis Institute, Jewish General Hospital, Montréal, Que.; The George Institute for Global Health (Jun), University of New South Wales, Sydney, Australia; ICES Central (Paterson); Institute of Health Policy, Management and Evaluation (Paterson), University of Toronto, Toronto, Ont.; Health Quality Council (Quail) and Department of Community Health and Epidemiology (Quail), University of Saskatchewan, Saskatoon, Sask.; Cardiology Service (Matteau), CHUM and CHUM Research Centre, Montréal, Que.

Contributors: Madeleine Durand and Mireille Schnitzer developed the study protocol and oversaw the creation of a data-analysis plan that was adapted for use in each jurisdiction and for each data set. Alexis Matteau contributed to the study design. Madeleine Durand drafted the manuscript, and Alexis Matteau revised the first draft. All of the authors contributed to study conception and protocol development, oversaw data analysis in each jurisdiction, contributed to data interpretation, revised the manuscript critically for important intellectual content, approved the final version to be published and agreed to be accountable for all aspects of the work.

Canadian Network for Observational Drug Effect Studies Investigators: Samy Suissa (principal investigator), Colin R. Dormuth (British Columbia), Brenda R. Hemmelgarn (Alberta), Gary F. Teare and Jacqueline Quail (Saskatchewan), Patricia Caetano and Dan Chateau 
(Manitoba), David A. Henry and J. Michael Paterson (Ontario), Jacques LeLorier (Quebec), Adrian R. Levy (Atlantic: Nova Scotia, Newfoundland and Labrador, New Brunswick, Prince Edward Island), Pierre Ernst and Kristian B. Filion (UK Clinical Practice Research Datalink), Robert W. Platt (methods) and Ingrid S. Sketris (knowledge translation).

Funding: The Canadian Network for Observational Drug Effect Studies, a collaborating centre of the Drug Safety and Effectiveness Network, is funded by grant DSE-146021 from the Canadian Institutes of Health Research (CIHR). Madeleine Durand is supported by a salary support award from the Fonds de recherche du Québec - Santé (FRQS). Mireille Schnitzer is supported by a New Investigator salary award from CIHR. Kristian Filion is supported by a salary support award from the FRQS and a William Dawson Scholar award from McGill University. Min Jun is supported by a Scientia Fellowship from UNSW Sydney, Australia. Christel Renoux is supported by a salary support award from the FRQS.

Data sharing: Access to data used in this study is under the jurisdiction of each site and with ethical requirements that are specific for each site and each data base used in the study. Data were not pooled across sites in this work. As such, any request for data access must be done on a site-bysite basis and will be subject to distinct procedures for each site. The full analytical protocol and underlying analytic codes (which are specific to each site) are available from the corresponding author on request.

Acknowledgements: This study was made possible through data-sharing agreements between Canadian Network for Observational Drug Effect Studies (CNODES) member research centres and the provincial governments of British Columbia, Alberta, Saskatchewan, Manitoba (Health Information Privacy Committee 2017/2018-07), Ontario, Quebec and Nova Scotia. This study was approved by the Independent Scientific Advisory Committee (protocol 17_101) of the UK Clinical Practice Research Datalink (CPRD). The final manuscript was approved by the CNODES Publications Committee. The BC Ministry of Health and BC Vital Statistics Agency approved access to and use of BC data facilitated by Population Data BC for this study. British Columbia data sources were as follows (https://www2.gov.bc.ca/gov/content/health/conducting -health-research-evaluation/data-access-health-data-central). British Columbia Ministry of Health [creator] (2017): Medical Services Plan (MSP) Payment Information File. BC Ministry of Health [publisher]. MOH (2017); British Columbia Ministry of Health [creator] (2017): PharmaNet. BC Ministry of Health [publisher]. Data Stewardship Committee (2017); Canadian Institute for Health Information (CIHI) [creator] (2017): National Ambulatory Care Reporting System. BC Ministry of Health [publisher]. MOH (2017); CIHI [creator] (2017): Discharge Abstract Database (Hospital Separations). BC Ministry of Health [publisher]. MOH (2017); British Columbia Ministry of Health [creator] (2017): Consolidation File (MSP Registration \& Premium Billing). BC Ministry of Health [publisher]. MOH (2017); BC Vital Statistics Agency [creator] (2017): Vital Statistics Deaths. BC Ministry of Health [publisher]. Vital Statistics Agency (2017). The authors acknowledge the programming support of Jianguo Zhang (Alberta), Rui Nie (CPRD), Yan Wang and Steve Doucette (Nova Scotia), Anjie Huang (Ontario), Matthew Dahl (Manitoba), Sarasa Johnson (Quebec), Nianping Hu (Saskatchewan) and Wusiman Aibibula (study coordinating centre). They also acknowledge the important contributions of the CNODES project manager, Corine Mizrahi, the research assistants, Carla Doyle and Carolina Moriello, and the CNODES collaborators and assistants at each site. The authors acknowledge Liliya Sinyavskaya, who provided pilot work with the Régie de l'assurance maladie du Québec database.

Disclaimer: Parts of this material are based on data or information compiled and provided by the Canadian Institute for Health Information (CIHI). The opinions, results and conclusions reported in this paper are those of the authors. No endorsement by the provinces, data stewards or $\mathrm{CIHI}$ is intended or should be inferred.

Supplemental information: For reviewer comments and the original submission of this manuscript, please see www.cmajopen.ca/content/8/4/ E877/suppl/DC1. 